\title{
A Survey on Image Mining, its Techniques and Application
}

\author{
Vaibhavi S. Shukla \\ M.E. Student \\ Department of Information Technology \\ GCET ,V .V .Nagar ,Affiliated to G.T.U. \\ Gujarat, India
}

\author{
Jay Vala \\ Assistant Professor \\ Department of Information Technology \\ GCET ,V .V .Nagar ,Affiliated to G.T.U. \\ Gujarat, India
}

\begin{abstract}
We know that today's world is digital world and we have use digital data such as video, audio, images etc. in various fields for various purposes. In present scenario, image plays vital role in every aspect of business such as business images, satellite images, and medical images and so on. Image mining is challenging field which extends traditional data mining from structured data to unstructured data such as image data. The main aim of this paper is to present a survey of the various techniques used for image mining applications. Forests fires are a significant problem. To fight against these disasters, the accurate prediction of forest fire is a crucial issue. The increase in the number of forest fires in the last few years has forced governments to take precautions. If the fire fighters know where the fire will be in sometimes it would be easier for them to stop the fire. Therefore a big need for predicting the fire behavior exists. In this paper various techniques of image mining and different algorithms used to analyze a key event -fire is studied. This paper covers literature survey of image mining techniques and its applications.
\end{abstract}

\section{Keywords}

Data mining, Multimedia mining, Image mining, forest fire

\section{INTRODUCTION}

Multimedia data mining is used for extracting interesting information for multimedia data sets, such as audio, video, images, graphics, speech, text and combination of several types of data set which are all converted from different formats into digital media [1]. As in day to day life importance associated with images is improving, Image Mining has became an important research issue in the area of Multimedia Mining. Research in Image mining can be broadly classified in two main directions (1) Domain specific applications (2) General applications[2].Image Mining is different from computer vision, and image processing techniques. This is because the focus of image mining is in the extraction of patterns from a large collection of images, whereas the focus of computer vision and image processing techniques is in understanding and/or extracting specific features from a single image [3]. Variety of image data for instance digital photographs, medical images and satellite TV images are generally generated each day. It is difficult or even impossible pertaining to human to learn the underlying knowledge and patterns inside the image when handling a sizable collection associated with images. Current research in image mining continues to be focused on how best to represent images so that data mining techniques can be applied [4]. Image mining is used in variety of fields like medical diagnosis, space research, remote sensing, satellite images, agriculture, and industries and even in the educational field [5].
Throughout Image mining procedure includes some steps like first refining Image database, Pre-processing, Transformation and have Extraction, Mining, Interpretation and at long last Evaluation that determined by knowledge. Here, images from an image database are first pre-processed to raise their quality. These images subsequently undergo various transformations and have extraction to generate giving her very features from the actual images. With the actual generated features, mining can be executed using data mining processes to discover significant styles. The resulting styles are evaluated and interpreted to discover the final knowledge, which may be applied to purposes.

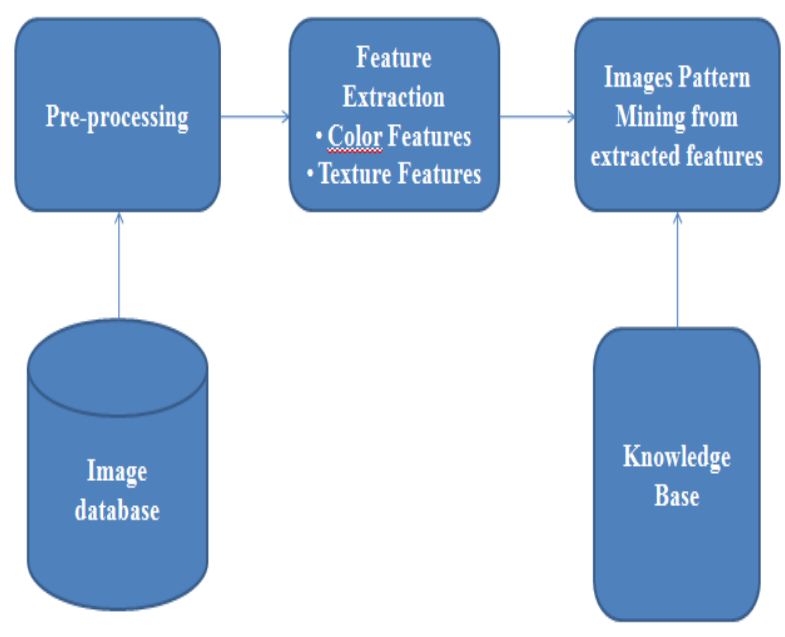

Fig.1. Image Mining Process

\section{IMAGE MINING FRAMEWORKS}

At the moment, a couple types of frameworks work extremely well: (a) Function -Driven Structure (b) Information-Driven image Frameworks [6]. A lot of current image mining process architectures come under this function-driven image mining framework. These descriptions are usually entirely application-oriented and also the framework has been arranged In line with the module performance. While the function-driven framework provides the goal of setting up and clarifying the various roles and duties to become carried out throughout image mining, that fails to emphasize the various amounts of Information representation needed for image facts prior to meaningful mining usually takes location [4].

\section{NEED OF IMAGE MINING}

Images, if analyzed, can reveal useful information towards the human users. Image mining refers to the extraction regarding implicit knowledge, image data relationship, or other designs not explicitly stored inside images. Image 
mining is over just an extendable of data mining to image sector.

\section{IMAGE MINING TECHNIQUES}

Image mining includes object recognition, image indexing and retrieval, image classification and clustering, association rules mining, and neural network $[6,7]$.

\subsection{Object Recognition}

Using object models which might be known a priori, an object recognition technique finds objects in actuality from an image. Machine learning and purposeful information extraction can simply be realized when some objects have been identified and recognized through machine. The object recognition problem might be refer to as any supervised labelling problem according to models of known items i. e. given a target image containing a number interesting objects and a collection of labels corresponding to a collection of models known to technique, what is object recognition to assign correct product labels to regions, or a collection of regions, in the image.

\subsection{Image Retrieval}

Image mining requires that images be retrieved according to some requirement specifications. The requirement specifications can be classified into three levels of increasing complexity:

(a) Level 1 comprises low level features of such as color, texture, shape or the spatial location of image elements.

(b) Level 2 comprises image retrieval by derived or logical features like objects of a given type or individual objects or persons.

(c) Level 3 comprises high level features of image.

\subsection{Image Indexing}

To further improve image retrieval rate, there is require of image data base using a fast and useful indexing scheme. A couple of main approaches are usually: reducing dimensionality or indexing high dimensional info. Other proposed indexing schemes concentrate on specific image features including color, shape and texture features.

\subsection{Image Classification}

In supervised classification technique, as input a collection of labelled (Pre-classified) images are given, and here the problem is to label a newly Encountered, yet unlabeled images. Typically, the given Labelled (training) images are used to do the machine learning of the class description which in turn is use to label a new Image.

\subsection{Image Clustering}

In unsupervised classification (or image clustering), the problem is always to group a given assortment of unlabeled images straight into Meaningful clusters based on the image content with not a priori knowledge. Clustering is often more advantage for minimizing the searching time period of images inside database. There are a variety of clustering methods: hierarchal, partitioning, density-based, grid based and fuzzy clustering methods.

\subsection{Association rules mining}

Association rule mining generates rules who have support and confidence greater than some user specific minimum support in addition to minimum confidence thresholds. A normal association rule mining algorithm works within two steps. The 1 st step finds all substantial item sets that match the minimum support constraint. The second move generates rules from each of the large item sets that match the minimum confidence constraint.

\subsection{Neural network}

Neural Networks are computational systems made up of simple processing units called neurons which are usually organized into layers with fully or partially connections. The main task associated with a neuron is to receive the activation values from its neighbours (the output of other neurons), compute an output based on its weighted input parameters and send that output to its neighbours.

\section{IMAGE MINING APPLICATIONS}

Image mining is use in various fields. Different applications of image are; $[2,3,5,6]$

- In medical for diagnose diseases.(e.g. Brain tumour)

- $\quad$ Satellite Cloud Imagery (e.g. Detecting copying unauthorized image on internet)

- In Natural scene recognition

- In Space research

- In Remote sensing

- In Detection of wild plant(e.g. egeria detection)

- In Agriculture field

- In industrial work

- In educational field

Image Mining Real-World Application [8]: It involves satellite images. Satellite images are a significant source of information. One useful request of satellite images is usually to examine the walkways and trends of forest fires over the years, thereby enabling fire-fighters to get a Better understanding of the behaviour of like forest fires so as to combat these that will fire effectively. This is aim for the satellite image mining application. To achieve this, following is required:

1. An efficient as well as effective spatial clustering technique for large-scale multi-resolution incremental clustering which might be adaptable in vibrant environment;

2. An image indexing scheme depending on clusterrelated semantic concepts to obtain high-level image retrieval within the satellite image database;

3. Fire cluster information to find out any spatial as well as temporal trends as well as patterns of fire development in terms of scale, area, time duration and location.

Different parameters for forest fire are:

- To detect affected region from forest fire, fire flames, fire behaviour from wind direction, prediction of fire spread [9].

- Identify fire spread path, to predict areas which may be affected in later stage, loss of vegetation and loss of wild life [10].

- Temperature extraction for fire occurrence, to identify fire occurring temperature, fire danger rate for loss of vegetation [11]

- Identify forest fire based on color space [12]. 
- Graph based forest fire detection which detects fire pixels [13].

- Relationship between size of forest fire and meteorically variables like temperature, relative humidity, wind speed, rainfall. In future enhancement, topological factors, types of forest and location [14]

- Predict/forecast/foresee/envisage and analysis of air pollution caused by forest fire, analysis of air quality in fire affected place [15].

- To estimating burned area, to map and assess burned areas from systematic sample of medium resolution satellite data [16].

- To develop model for monitoring and analysing forest fires in space and time, predict motion of fire [17].

\section{IMAGE MINING ISSUES}

Image mining research remains in their infancy and many issues continue to be solved. Particularly, for image mining research to progress to a fresh height, the pursuing issues need to be investigated.

Issues [8]:

(a) Propose new representation schemes for visual patterns that are able to encode sufficient contextual information to allow for meaningful extraction of useful visual characteristics.

(b) (b) Devise efficient content-based image indexing and retrieval techniques to facilitate fast and effective access in large image repository.

(c) Design semantically powerful query languages for image databases;

(d) Explore new discovery techniques that take into account the unique characteristics of image data;

(e) Incorporate new visualization techniques for the visualization of image patterns.

(f) Central key issue in image mining is how to preprocess image sets so as to represent in form that supports the application of data mining.

(g) Image pattern representation: How can we represent the image pattern such that the contextual information, spatial information, and important image characteristics are retained in the representation scheme?

(h) Image features selection: Which are the important images features to be used in the mining process so that the discovered patterns are meaningful visually?

(i) Image pattern visualization: How to present the mined patterns to the user in a visually-rich environment?

\section{CONCLUSION}

The main intent of the image mining is to remove the data loss and obtain the meaningful information which is expected need of human. In this paper most of image mining techniques have been discussed. These all techniques have their own advantages and disadvantages. For particular forest fire application, by using more than one number of parameters to predict forest fire may give better result. They use different methods for the same.

\section{REFERENCES}

[1] Dr. S. Vijayarani and Ms. A. Sakila, "Multimedia Mining Research - An Overview," in International Journal of Computer Graphics \& Animation (IJCGA) Vol.5, No.1, January 2015.

[2] A.Hema, E. Annasaro, "A Survey In Need Of Image Mining Techniques," in International Journal of Advanced Research in Computer and Communication Engineering Vol. 2, Issue 2, February 2013.

[3] A. A. Khodaskar, S .A. Ladhake "Image Mining: An Overview of Current Research," in Fourth International Conference on Communication Systems and Network Technologies, IEEE 2014

[4] T.Karthikeyan, P.Manikandaprabhu, "Function and Information Driven Frameworks for Image Mining - A Review" in International Journal of Advanced Research in Computer and Communication Engineering Vol. 2, Issue 11, November 2013.

[5] Deepika Kishor Nagthane, "Image Mining Techniques and Applications", in International Journal Of Engineering Sciences \& Research Technology July, 2013.

[6] K. R. Yasodha, K.S. Yuvaraj, "A Study on Image Mining Techniques", In International Journal of Applied, December 2013.

[7] Prabhjeet Kaur, Kamaljit Kaur, " Review of Different Existing Image Mining Techniques”, In International Journal of Advanced Research in Computer Science and Software Engineering, June 2014.

[8] Ji Zhang, Wynne Hsu, Mong Li Lee, "Image Mining: Trends and Developments", International Workshop on Multimedia Data Mining, 2001.

[9] Divya T.L, Vijayalakshmi M.N, "Analysis of Wild Fire Behaviour in Wild conservation Area using Image Data mining”,,IEEE,2015

[10] Divya TL, Vijayalakshmi M.N, "Development of Frame Work for Prediction of Forest Fire and Fire Spread Direction Using Image Mining", IJARCCE, 2013

[11] Divya TL, Vijayalakshmi M.N, "Implementation of data mining techniques for Temperature Extraction for forest fire occurrence with Image Analysis", IJIRCCE,2013

[12] Fei Van, Xing Xu, Ning Han, "Identification Method of Forest Fire Based on Color Space”,,IEEE,2010

[13] Young Gi Byun, Yong Huh, Kiyun Yu, Yong Il Kim, "Evaluation of Graph-based Analysis for Forest Fire Detections”,IEEE,2005

[14] Yong Poh Yu, Rosli Omar,Rhett D. Harrison, Mohan Kumar Sammathuria and Abdul Rahim Nik," Pattern clustering of forest fires based on meteorological variables and its classification using hybrid data mining methods" Journal of Computational Biology and Bioinformatics Research, 2011 
[15] Divya TL, Dr.Vijayalakshmi MN, "Envisagation and Analysis of Air Pollution Caused by Forest fire using Machine Learning Algorithm”, IRJET ,2015

[16] Yosio Edemir Shimabukuro, Jukka Miettinen, René Beuchle, Rosana Cristina Grecchi,Dario Simonetti, and Frédéric Achard, "Estimating Burned Area in Mato Grosso, Brazil,Using an Object-Based Classification
Method on a Systematic Sample of Medium Resolution Satellite Images”,IEEE,2015

[17] Rajasekar Umamaheshwaran, Wietske Bijker, and Alfred Stein, "Image Mining for Modeling of Forest Fires From Meteosat Images", IEEE Transactions On Geoscience And Remote Sensing, Vol. 45, No. 1, January 2007. 